21. McCune B., Grace J.B. Analysis of Ecological Communities. MjM SoftWare Design, 2002. 300 p.

22. McLachlan G.J. Discriminant analysis and statistical pattern recognition. Wiley-Interscience: Hoboken, 2004. $580 \mathrm{p}$.

23. Persson S. Ecological indicator values as an aid in the interpretation of ordination diagrams // Journal of Ecology. 1981. Vol. 69, № 1. P. 71-84.

\title{
PATTERNS IN GRASSLAND VEGETATION OF BREDINSKIY STATE NATURAL WILDLIFE PRESERVE
}

(C) 2018

Nazarenko Nazar Nikolayevich, doctor of biological sciences, professor of Chemistry, Ecology and Chemistry Methodology Department

Perlov Yevgeny Dmitrievich, student of Natural Sciences and Technologies Faculty South Ural State Humanitarian Pedagogical University (Chelyabinsk, Russian Federation)

Abstract. The patterns (mosaicity) in grassland vegetation of Southern Ural for Bredinskiy state natural wildlife preserve example have been characterized by a hierarchical continuum concept theory. The multispecies patterns identification has been done by blocks and principal components methods and hierarchical patterns evaluation - by multivariate statistics (Cluster, Discriminant Analysis and Non-Metric Multidimensional Scaling) and biotopes phytoindication. It has been established that there are two patterns levels - parcels $(6,0 \mathrm{~m})$ and coenotics $(11,0 \mathrm{~m}) \mathrm{in}$ grassland vegetation. Thus, hierarchical continuum in steppe grassland vegetation of Southern Ural has been confirmed by multivariate statistics. Detected patterns have been classified and its phytochorologic unit has been identified. For patterns informative and dominant species have been also detected. It has been demonstrated that species dominance and its significance in patterns depend upon mosaicity levels. Some non-uniformly scaled ecological factors have been identified for patterns continuum. The principal significance for steppe grassland parcels matter coenotic and phytodiversity factors, for coenotics patterns forming matter abiotic factors too. For Bredinskiy state natural wildlife preserve principal abiotic factors steppe grassland patterns forming are (by decrease) soil calcium regime, soil nitrogen regime, soil acidity, soil mineralization and aeration.

Keywords: hierarchical continuum; mosaicity; patterns; microsites; parcels; coenosis; biotopes; phytochorologic units; abiotic factors; ordination; phytoindication; steppe grassland vegetation of South Ural; Bredinskiy state natural wildlife preserve; Chelyabinsk Region.

УДК 581.5

DOI 10.24411/2309-4370-2018-14116

Статья поступила в редакцию 25.09.2018

\section{ПРОСТРАНСТВЕННАЯ ОРГАНИЗАЦИЯ ПОПУЛЯЦИЙ} CYCLACHAENA XАNTHIIFOLIA (NUTT.) FRESEN. В ПОЛОСЕ ОТВОДА ЖЕЛЕЗНЫХ ДОРОГ (C) 2018

Никитин Николай Александрович, аспирант кафедры биологии, экологии и методики обучения Самарский государственный соииально-педагогический университет (2. Самара, Российская Федераиия)

Аннотащия. Пространственная структура популяций в условиях антропогенного воздействия является очень важным показателем, отражающим поведенческие паттерны вида как изменение структуры и агрегированности скоплений особей. Карантинные сорняки несут наибольшую опасность населению, поскольку чаще всего оказываются причиной поллинозов и значительного ухудшения качества жизни во время сезонных вспышек заболевания. Являясь объектом пристального внимания экологов, они должны быть исследованы в первую очередь методами анализа пространственной структуры их популяций. Железные дороги и полосы отвода считаются коридорами, по которым карантинные сорняки и другие чужеродные и инвазионные виды способны расселяться на новые территории. Пространственное поведение видов, образование агрегаций и больших скоплений либо равномерное расселение особей может раскрыть фундаментальные взаимосвязи между условиями антропогенного воздействия в пределах полосы отвода и пространственной структурой популяций. Исследования показали, что в пределах полосы отвода железных дорог перегона КинельБезенчук Куйбышевской железной дороги в пространственной структуре популяции Cyclachaena xanthiifolia (Nutt.) Fresen. наблюдается групповое и равномерное распределение особей. Агрегация особей в скопления (групповое распространение) тяготеет к местам с наибольшей антропогенной нагрузкой, что подтверждается показателями ранговой корреляции.

Ключевые слова: железные дороги; полоса отвода железных дорог; индекс Одума; индекс Морисита; пространственная структура популяций; популяции; абсолютная плотность популяции; ранговая корреляция; Cyclachaena xanthiifolia (Nutt.) Fresen.; антропогенный фактор; агрегации особей; пространственная гетерогенность; метод пробных площадок; коридоры расселения; инвазионные виды; чужеродные виды; карантинные сорняки. 
Пространственная организация популяции является одной из важнейших характерных особенностей, позволяющих оценить ее структуру, факториальные особенности среды, характер распространения отдельных особей в пространстве, а также дисперсионную агрегативность вида в целом [1-10]. Так, в работах одного из крупнейших американских экологов Роберта Уиттекера поведенческие паттерны отдельных видов раскрываются через понятия жизненных стратегий или эколого-ценотических стратегий, согласно которым каждый вид в ответ на определенные условия окружающей среды вырабатывает специфический комплекс приспособленческих механизмов, позволяющих в данных условиях сохранять численность, занимать новые экологические ниши, так или иначе поддерживая гомеостаз популяции.

Важным показателем пространственной структуры является пространственная гетерогенность, основывающаяся на выборочных данных о численности особей в пределах пробных площадок. Ряд ученых указывает на ту или иную пространственную организацию определенных видов как характерную особенность, присущую только им, и как неотъемлемую особенность, сформировавшуюся в ходе филогенеза таксона [11-17]. Основоположник экологической научной школы США Юджин Одум в своих работах писал об особенностях формирования растительных сообществ Великих равнин и вегетативного способа размножения некоторых злаков, отмечая при этом характерные для данных видов форму и структуру популяций [18]. Также на это указывают видные ученые из Великобритании Дэннис Тейлор, Уилф Стаут и Найджел Грин, уделяя особое внимание популяциям растений, распространенных в литоральных и сублиторальных областях побережья Атлантического океана. Формируемые популяции характеризовались определенной линейной структурой [3]. Изучая кустарниковую растительность, сформировавшуюся в условиях средиземноморского климата южного побережья штата Калифорния, а также растительность горных областей центральных штатов США, Роберт Уиттекер отмечал схожие тенденции в формировании пространственной структуры популяций, при совпадении почвенно-геологических условий $[5 ; 12]$.

Воздействие антропогенного фактора на пространственную структуру популяций рассматривается в работах тех же ученых. Однако детального рассмотрения влияния железнодорожной инфраструктуры, как одного из основных видов транспорта на планете, не встречается, либо оно указывается как часть обобщенного антропогенного воздействия. Антропогенное воздействие посредством железных дорог, в свою очередь, приводит к характерным изменениям в структуре популяций, которые могут и должны быть отслежены, измерены и статистически интерпретированы.

Особый интерес представляет влияние антропогенного фактора на пространственную структуру популяций карантинных сорняков, представляющих угрозу здоровью населения. Выявление тех или иных особенностей, появляющихся в пространственной структуре как результирующее воздействие антропогенных факторов, представляется весьма актуальной задачей в русле исследований популяций видов, рас- пространяющихся в условиях антропогенного воздействия. Полученные данные позволят прогнозировать поведение видов в условиях антропогенного пресса и разрабатывать адекватные программы противодействия инвазиям.

\section{Материал и методы}

Исследования пространственной структуры популяций Cyclachaena xanthiifolia (Nutt.) Fresen. проводились в Волжском районе Самарской области, на перегоне Кинель-Безенчук Куйбышевской железной дороги в окрестностях села Николаевка в 2018 году. Выбранная площадка является местом постоянного мониторинга эколого-ботанических и флористических особенностей полосы отвода железных дорог на протяжении 8 лет.

Основой проводимых исследований является сеть пробных площадок, на которые был разбит изучаемый район. Пробные площадки располагались вдоль следования железнодорожного полотна, в шахматном порядке. В пределах каждой из них фиксировалось общее количество произрастающих особей исследуемого вида, а также уровень антропогенной нагрузки по шкале от 1 до 3: 1 - значительное антропогенное воздействие, 2 - среднее и 3 - незначительное. Данная градация удобна для применения в полевых условиях, а также в дальнейшем при проведении статистических расчетов.

В ходе исследований применялись методы изучения пространственной структуры популяций, основанные на однократном учете, а именно индексы Одума и Морисита.

Индекс Одума $\left(I_{o}\right)$ разработан одним из основоположников современной экологии американским ученым Юджином Одумом [18].

$$
I_{o}=\frac{S^{2}}{D}
$$

Данный индекс основан на математической особенности случайного распределения, для которого среднее арифметическое равно квадрату стандартного отклонения.

$$
D=\frac{\sum x_{i}}{n}
$$

Определив абсолютную плотность организмов $(D)$ как среднее арифметическое зарегистрированных в пределах каждой пробной площадки $\left(\mathrm{x}_{\mathrm{i}}\right)$ и рассчитав квадрат стандартного отклонения, получим путем расчета индекса Одума определенное значение, уровень значимости которого проверяется путем сравнения с табличным значением F-критерия Фишера-Снедекора с числом степеней свободы $d f_{1}=d f_{2}=n-1$. При $\mathrm{I}_{\mathrm{o}}<1-$ особи в популяции распределены равномерно, $\mathrm{I}_{\mathrm{o}}>1$ - распределение групповое, $\mathrm{I}_{\mathrm{o}}=1$ - распределение особей в популяции случайное.

Одним из недостатков указанного метода является допущение того, что изучаемая выборка меньше, чем скопления, образованные особями.

Лишенным этого недостатка является индекс, позволяющий анализировать выборки любых размеров, собранных на любых размеров пробных площадках. Таким индексом является индекс Морисита $[19 ; 20]$.

$$
I_{s}=n \frac{\sum_{i-1}^{n} N_{i}\left(N_{i}-1\right)}{N(N-1)}
$$

Самарский научный вестник. 2018. Т. 7, № 4 (25) 
Интерпретация полученных результатов заключается в том, что при $\mathrm{I}_{\delta}=1$ наблюдается случайное расселение, $\mathrm{I}_{\delta}<1$ равномерное, $\mathrm{I}_{\delta}>1$ групповое.

В ходе исследования было выделено 20 пробных площадок $\mathrm{S}=1 \mathrm{M}^{2}$. Все они располагались в полосе отвода железных дорог. В границах 1, 3, 4, 5, 7, 10, 18, 19, 20 пробных площадок обнаруживались следы активного антропогенного воздействия, особи произрастали на недавно отремонтированной насыпи из щебня, а также на свалах строительного мусора и щебня, оставшихся после ремонта железнодорожного полотна. Наблюдения проводились в июле 2018 г. в период активной вегетации исследуемого вида.

Таблица 1 - Пробные площадки в полосе отвода железной дороги на перегоне Кинель-Безенчук Куйбышевской железной дороги

\begin{tabular}{|c|c|c|c|}
\hline \multirow{2}{*}{$\begin{array}{c}\text { Порядковый } \\
\text { номер } \\
\text { площадки }\end{array}$} & \multirow{2}{*}{$\begin{array}{c}\text { Площадь, } \\
\text { м }^{2}\end{array}$} & \multicolumn{2}{|c|}{ Координаты } \\
\hline & & $\begin{array}{l}\text { широта, } \\
\text { ○ с.ш. }\end{array}$ & $\begin{array}{c}\text { долгота, } \\
\text { о в.д. }\end{array}$ \\
\hline 1 & 1 & 53,085220 & 50,325246 \\
\hline 2 & 1 & 53,085581 & 50,324470 \\
\hline 3 & 1 & 53,085442 & 50,325332 \\
\hline 4 & 1 & 53,085994 & 50,324642 \\
\hline 5 & 1 & 53,087249 & 50,326602 \\
\hline 6 & 1 & 53,087184 & 50,327164 \\
\hline 7 & 1 & 53,087521 & 50,326991 \\
\hline 8 & 1 & 53,087521 & 50,327498 \\
\hline 9 & 1 & 53,087735 & 50,327207 \\
\hline 10 & 1 & 53,087735 & 50,327714 \\
\hline 11 & 1 & 53,088098 & 50,327584 \\
\hline 12 & 1 & 53,088053 & 50,327993 \\
\hline 13 & 1 & 53,088286 & 50,327874 \\
\hline 14 & 1 & 53,088280 & 50,328304 \\
\hline 15 & 1 & 53,088545 & 50,328078 \\
\hline 16 & 1 & 53,088526 & 50,328593 \\
\hline 17 & 1 & 53,088430 & 50,328324 \\
\hline 18 & 1 & 53,088849 & 50,328978 \\
\hline 19 & 1 & 53,089154 & 50,328700 \\
\hline 20 & 1 & 53,089115 & 50,329306 \\
\hline
\end{tabular}

\section{Результаты исследований} и их обсуждение

В ходе исследования были извлечены выборки, рассчитаны необходимые параметры для расчета индексов.

Анализируя полученные результаты вычисления $\mathrm{I}_{\mathrm{o}}$ можно сделать вывод о групповом распределении особей Cyclachaena xanthiifolia (Nutt.) Fresen. в популяции, произрастающей в полосе отвода железных дорог перегона Кинель-Безенчук Куйбышевской железной дороги. Учитывая постоянное антропогенное воздействие, можно констатировать тот факт, что именно его воздействие привело к группированию агрегаций особей и, как следствие, групповой пространственной структуре популяции.

Значение $\mathrm{I}_{\delta}$, как более чувствительного к агрегированности особей и малозависимого от размера выборки и площади пробной площадки показало заметную разницу в показателях. В пределах десяти пло- щадок обнаружилось равномерное распределение особей (рис. 2), в восьми групповое (рис. 1), в пределах одной площадки особей исследуемого вида обнаружено не было и в пределах одной площадки особи были распространены случайно.

Таблица 2 - Результаты расчета основных показателей пространственной структуры популяции Сусlachaena xanthiifolia (Nutt.) Fresen. в полосе отвода железных дорог перегона Кинель-Безенчук Куйбышевской железной дороги

\begin{tabular}{|c|c|c|c|c|c|c|}
\hline $\begin{array}{c}\text { № пло- } \\
\text { щадки }\end{array}$ & $\mathrm{S}^{2}$ & $\mathrm{~F}_{\mathrm{A}}$ & $\mathrm{X}_{\mathrm{i}}$ & $\mathrm{D}$ & $\mathrm{I}_{\mathrm{o}}$ & $\mathrm{I}_{\delta}$ \\
\hline 1 & 647 & 1 & 21 & 19 & $34(1,77 *)$ & 2,157 \\
\hline 2 & 647 & 2 & 6 & 19 & $34(1,77 *)$ & 0,578 \\
\hline 3 & 647 & 2 & 8 & 19 & $34(1,77 *)$ & 0,789 \\
\hline 4 & 647 & 1 & 85 & 19 & $34(1,77 *)$ & 9,000 \\
\hline 5 & 647 & 1 & 78 & 19 & $34\left(1,77^{*}\right)$ & 8,157 \\
\hline 6 & 647 & 2 & 5 & 19 & $34(1,77 *)$ & 0,473 \\
\hline 7 & 647 & 1 & 65 & 19 & $34(1,77 *)$ & 6,789 \\
\hline 8 & 647 & 2 & 2 & 19 & $34(1,77 *)$ & 0,157 \\
\hline 9 & 647 & 3 & 10 & 19 & $34(1,77 *)$ & 1,000 \\
\hline 10 & 647 & 1 & 0 & 19 & $34(1,77 *)$ & - \\
\hline 11 & 647 & 2 & 5 & 19 & $34\left(1,77^{*}\right)$ & 0,578 \\
\hline 12 & 647 & 1 & 15 & 19 & $34(1,77 *)$ & 1,526 \\
\hline 13 & 647 & 2 & 9 & 19 & $34(1,77 *)$ & 0,894 \\
\hline 14 & 647 & 2 & 3 & 19 & $34\left(1,77^{*}\right)$ & 0,263 \\
\hline 15 & 647 & 1 & 8 & 19 & $34\left(1,77^{*}\right)$ & 1,421 \\
\hline 16 & 647 & 2 & 7 & 19 & $34(1,77 *)$ & 0,789 \\
\hline 17 & 647 & 2 & 27 & 19 & $34(1,77 *)$ & 0,648 \\
\hline 18 & 647 & 1 & 11 & 19 & $34\left(1,77^{*}\right)$ & 2,789 \\
\hline 19 & 647 & 1 & 7 & 19 & $34(1,77 *)$ & 1,105 \\
\hline 20 & 647 & 2 & 2 & 19 & $34(1,77 *)$ & 0,684 \\
\hline
\end{tabular}

Примечание. *Согласно таблице значений F-критерия Фишера-Снедекора для $d f_{1}=d f_{2}=n-1$.

Предваряя анализ полученных данных, были сформулированы рабочие гипотезы $\left(\mathrm{H}_{1}\right)$ :

1. В полосе отвода железных дорог среди популяций Cyclachaena xanthiifolia (Nutt.) Fresen. преобладает групповое распределение особей.

2. Между групповым (агрегированным) распределением особей и степенью антропогенного воздействия существует статистически значимая корреляция.

Для доказательства первой гипотезы использовался метод углового преобразования Фишера ( $\varphi$ критерий). Расчетное значение данного показателя оказалось в зоне незначимости $\varphi_{э м п}=0,395$, что отвергло $\mathrm{H}_{1}$, лишив нас права утверждать, что групповое распределения особей Cyclachaena xanthiifolia (Nutt.) Fresen. преобладает в полосе отвода железных дорог.

Для доказательства второй гипотезы применялся критерий ранговой корреляции Спирмена, расчетное значение которого оказалось в зоне значимости $\mathrm{R}_{\mathrm{s}}=-0,604$, что подтвердило $\mathrm{H}_{1}$ и позволило говорить о зависимости агрегированности особей и пробных площадок с различным уровнем антропогенной нагрузки, которая распределялась следующим образом: к пробным площадкам с повышенным уровнем антропогенной нагрузки тяготеют групповые скопления особей. 
Стоит отметить, что в ходе исследования распределение особей не всегда очевидно определялось методом визирования и осмотра пробных площадок, что еще раз подтверждает важность и актуальность использования методов математической статистики и методов изучения пространственной структуры популяций методами индексирования пространственной гетерогенности популяций. Перспективным видится и рассмотрение агрегаций с точки зрения половозрастного состава, стадий онтогенеза, сравнение данных показателей у чужеродных и аборигенных видов.

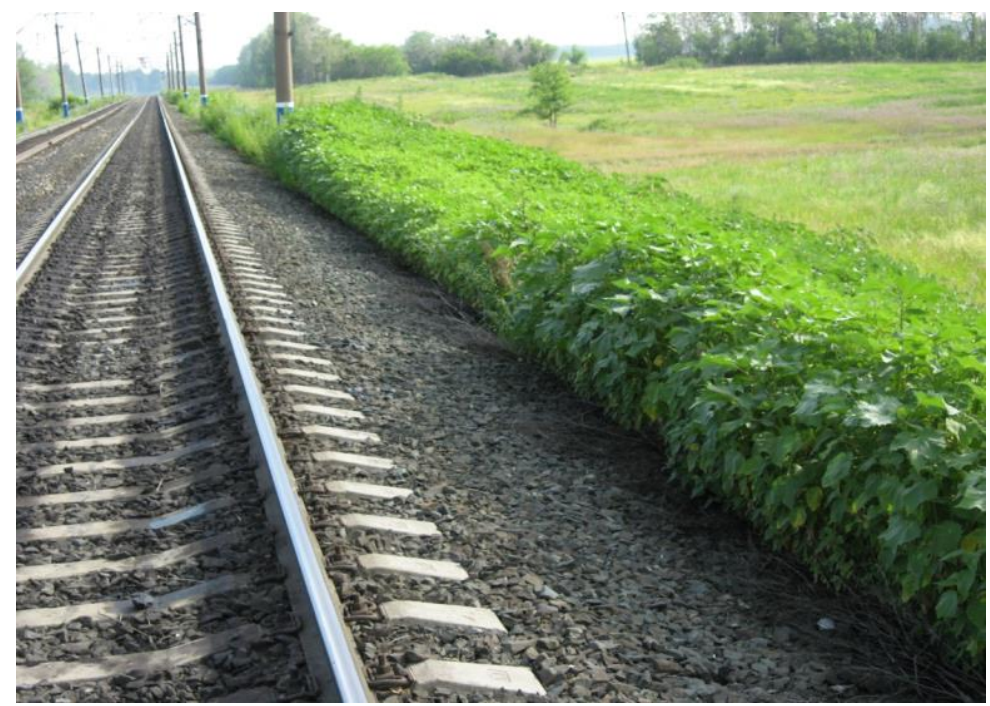

Рисунок 1 - Пробная площадка № 10, в границах которой было зафиксировано групповое произрастание особей

\section{Выводы}

В пределах полосы отвода железных дорог в пространственной структуре популяции Cyclachaena xanthiifolia (Nutt.) Fresen. наблюдается групповое $\left(\mathrm{I}_{\delta}>1 ; \mathrm{I}_{\mathrm{o}}>1\right)$ и равномерное распределение особей $\left(\mathrm{I}_{\delta}<1 ; \mathrm{I}_{0}<1\right)$, без преобладания какого-либо из них $\left(\varphi_{э м п}=0,395\right)$. Реакция вида на антропогенное воздействие путем образования агрегаций особей в скопления (групповое распространение) тяготеет к местам с наибольшей антропогенной нагрузкой $\left(\mathrm{R}_{\mathrm{s}}=-0,604\right)$.

\section{Список литературы:}

1. Iwao S. A new regression method for analyzing the aggregation pattern of animal populations // Researches on Population Ecology. 1968. Vol. 10, № 1. P. 1-20.

2. Taylor L.R. Aggregation, variance and the mean // Nature. 1961. Vol. 189. P. 732-735.

3. Тейлор Д., Грин Н., Стаут У. Биология: В 3-х т. Т. 2 / пер. с англ.; под ред. Р. Сопера 3-е изд. М.: Мир, 2004. 436 c.

4. Olsvig L.S., Cryan J.F., Whittaker R.H. Vegetational gradients of the pine plains and barrens of Long Island // Pine Barrens: Ecosystem and Landscape / Ed. by R.T.T. Forman. New York: Academic Press, 1979. P. 265-282.

5. Whittaker R.H. The ecology of serpentine soils. IV. The vegetational response to serpentine soils // Ecology. 1954. Vol. 35. P. 275-288.

6. Whittaker R.H. A criticism of the plant association and climatic climax concepts // Northwest Science. 1951. Vol. 25. P. 17-31.

7. Whittaker R.H. Gradient analysis of vegetation // Biological Reviews. 1967. Vol. 42. P. 207-264.

8. Whittaker R.H. Communities and Ecosystems. New York: Macmillan, 1975. 385 p. P. 284-307.

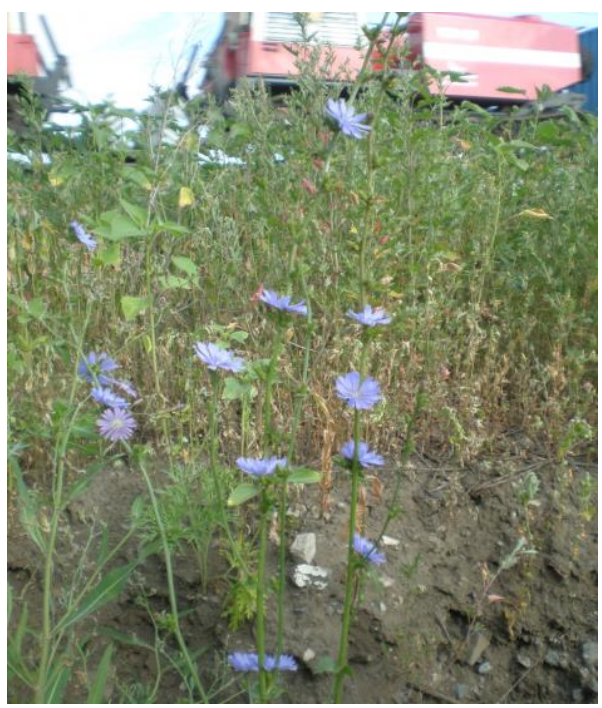

Рисунок 2 - Пробная площадка № 13, в пределах которой зафиксировано равномерное распределение особей

9. Whittaker R.H. Evolution and measurement of species diversity // Taxon. 1972. Vol. 21. P. 213-251.

10. Whittaker R.H., Likens G.E. The biosphere and man // Primary Productivity of the Biosphere / Ed. by H. Lieth, R.H. Whittaker. New York: Springer Verlag, 1975. P. 305-328.

11. Whittaker R.H. Introduction // Handbook of Vegetation Science. Part V: Ordination and Classification of Vegetation / Ed. by R.H. Whittaker. The Hague: Junk B.V., 1973. P. 1-6.

12. Shmida A., Whittaker R.H. Pattern and biological microsite effects in two shrub communities, southern California // Ecology. 1981. Vol. 62. P. 234-251.

13. Gauch H.G. Jr., Whittaker R.H. Hierarchical classification of community data // Journal of Ecology. 1981. Vol. 69. P. 537-557.

14. Tansley A.G. The use and abuse of vegetational concepts and terms // Ecology. 1935. Vol. 16, № 3.

15. Cliff A.D., Ord J.K. Spatial Processes. London: Pion, 1981. $266 \mathrm{p}$.

16. Soutwood T.R.E. Ecological methods. London: Chapman \& Hall, 1978. 524 p.

17. Elliot J.M. Some methods for the statistical analysis of samples of benthic invertebrates. Freshwater Biological Association, 1977. $160 \mathrm{p}$.

18. Одум Ю. Экология: В 2-х т. Т. 2 / пер. с англ. М.: Мир, 1986. 376 с.

19. Morisita M. Measuring the dispersion of individuals and analysis of the distributional patterns // Memoirs of the Faculty of Science, Kyushu University. Series E: Biology. 1959. № 2. P. 215-235.

20. Morisita M. $I_{\sigma}$-Index: a measure of dispersion of individuals // Researches on Population Ecology. 1962. Vol. 4, № 1. P. 1-7. 


\title{
SPATIAL ORGANIZATION OF CYCLACHAENA XANTHIIFOLIA (NUTT.) FRESEN. POPULATIONS ON THE RAILWAY LINE
}

\author{
(C) 2018 \\ Nikitin Nikolay Alexandrovich, postgraduate student of Chair of Biology, Ecology and Methods of Teaching \\ Samara State University of Social Sciences and Education (Samara, Russian Federation)
}

\begin{abstract}
The spatial structure of populations under the conditions of anthropogenic impact is a very important indicator that reflects the behavioral patterns of the species, such as changes in the structure and aggregation of individuals. Quarantine weeds are the object of ecologists' close attention, as these weeds carry the greatest danger to the population, since they most often cause pollinosis and a significant deterioration in the quality of life during seasonal outbreaks of the disease. That is why they should be investigated by methods of analyzing the spatial structure of their populations. Railways are considered to be corridors along which quarantine weeds and other alien and invasive species are able to spread to new territories. Spatial behavior of species, formation of aggregations and large clusters, or a uniform distribution of individuals can reveal a fundamental relationship between the conditions of anthropogenic impact along the right-of-way and the spatial structure of populations. Along the Kinel-Bezenchuk Kuybyshev railroad in the spatial structure of the population of Cyclachaena xanthiifolia (Nutt.) Fresen. there is a group and uniform distribution of individuals. Aggregation of individuals in clusters (group distribution) are found in the places with the highest anthropogenic load.

Keywords: railways; right-of-way; Odum index; Morisita index; spatial structure of populations; populations; absolute population density; rank correlation; Cyclachaena xanthiifolia (Nutt.) Fresen.; anthropogenic factor; aggregation of individuals; spatial heterogeneity; trial sites method; corridors of settlement; invasive species; alien species; quarantine weeds.
\end{abstract}

УДК 595.768.12

DOI 10.24411/2309-4370-2018-14117

Статья поступила в редакцию 16.10 .2018

\section{ФАУНА ЖУКОВ-ЛИСТОЕДОВ (COLEOPTERA, CHRYSOMELIDAE) ИЗОЛИРОВАННЫХ ВОЛЖСКИХ ОСТРОВОВ И МЕХАНИЗМЫ ИХ ВТОРИЧНОГО ЗАСЕЛЕНИЯ}

(C) 2018

Павлов Сергей Иванович, кандидат биологических наук, доцент кафедры биологии, экологии и методики обучения

Яицкий Андрей Степанович, старший преподаватель кафедры биологии, экологии и методики обучения Самарский государственный сочиально-педагогический университет (2. Самара, Российская Федераиия)

Аннотащия. В течение 1974-2016 гг. в природных и лабораторных условиях в Самарской области изучались особенности выживания имаго жуков-листоедов в результате паводкового разлива р. Волги, затопления рекой изолированных островных систем, а также - миграционные процессы насекомых после схода паводковой воды. За 12-летний период полевых исследований 11 специалистов-энтомологов, используя 2 традиционных и 6 авторских методов, на 11 относительно больших волжских островах (3 из которых периодически затапливаются) и 20 крупных, регулярно затапливаемых отмелях и косах собрали коллекцию из 29 видов жуков-хризомелид. Установлено, что фауна листоедов островов по генезису очень близка к фауне сопредельных побережий. Анализируется происхождение и рельеф типичных островов, уровень паводковых вод, продолжительность затопления поймы р. Волги близ Самарской Луки, механизмы выживания жуков путем адаптации их к условиям местообитаний или особенности их миграций, при вторичном заселении островных систем. Установлено, что, кроме активного перелета и автономного плавания на острова, жуки пользуются доступными «плавсредствами» - «плотами» из растительного паводкового шлама, мелкими и достаточно крупными плавающими объектами (от веток и досок до речных судов). Приведены данные по примерным долям участия разных способов передвижения имаго листоедов при их рассредоточении в пространстве. Установлено, что миграции происходят ежегодно, совершенно независимо от успеха перезимовки популяции островных жуков-предшественников.

Ключевые слова: жуки-листоеды; Coleoptera; Chrysomelidae; фауна; фауна жуков; приводные биотопы; островные биотопы; механизмы заселения биотопов; затопляемые острова; незатопляемые острова; песчаные отмели; песчаные косы; механизмы выживания в условиях подтопления; Среднее Поволжье; Самарская область; Волга; Самарская Лука; Жигулёвский заповедник.

\section{Памяти известного российского энтомолога, \\ Юрия Михайловича Зайцева, сотрудника \\ Института проблем экологии и эволюции}

им. А.Н. Севериова РАН (2. Москва), посвящается.

Фенологические особенности климата умеренных широт заключаются в значительной качественной трансформации температурных параметров окружа- ющей среды примерно в равных пределах $-25 \ldots$ $\ldots+25^{\circ} \mathrm{C}$. При низких температурах природа на 5,56 месяцев впадает в состояние глубокого покоя, а 